\title{
Exact Solution of the Curved Dirac Equation in Polar Coordinates: Master Function Approach
}

\author{
H. Panahi and L. Jahangiri \\ Department of Physics, University of Guilan, Rasht 41635-1914, Iran \\ Correspondence should be addressed to H. Panahi; t-panahi@guilan.ac.ir \\ Received 6 February 2015; Accepted 25 April 2015 \\ Academic Editor: Ming Liu
}

Copyright (C) 2015 H. Panahi and L. Jahangiri. This is an open access article distributed under the Creative Commons Attribution License, which permits unrestricted use, distribution, and reproduction in any medium, provided the original work is properly cited. The publication of this article was funded by SCOAP S $^{3}$

\begin{abstract}
We show that the $(2+1)$ curved Dirac equation in polar coordinates can be transformed into Schrodinger-like differential equation for upper spinor component. We compare this equation with the Schrodinger equation derived from shape invariance property of second order differential equations of mathematical physics. This formalism enables us to determine the electrostatic potential and relativistic energy in terms of master function and corresponding weight function. We also obtain the spinor wave function in terms of orthogonal polynomials.
\end{abstract}

\section{Introduction}

The Klein-Gordon and Dirac equations are relativistic wave equations which describe the behaviour of spin 0 and $1 / 2$ particles, respectively. These equations were formulated in order to construct a unified theory involving quantum mechanics and special relativity. Undoubtedly, the Dirac equation is one of the remarkable achievements in modern physics because of its important role in particle physics by predicting the existence of antiparticles. In order to investigate the relativistic effects, this equation has been solved for different potentials like Coulomb [1], Rosen-Morse [2], Woods-Saxon [3], generalized Poschl-Teller [4], Yukawa [5], and so forth. The Dirac equation was first written in Minkowski spacetime but in order to study the behaviour of fermions in gravitational fields, this equation has been extended to curved space-time. The Dirac equation in curved space-time has been considered in different curved backgrounds due to its considerable applications in astrophysics, cosmology, and condensed matter [6-9]. On the other hand, in recent years, different methods have been applied in order to study the Dirac equation such as separation of variables $[10,11]$, supersymmetric quantum mechanics [12,13], and NikiforovUvarov $[14,15]$. As we know the supersymmetry [16] and shape invariance [17] formalism is one of the most important methods which has been successfully used in order to study exactly solvable quantum systems. In this formalism, two partner Hamiltonians can be factorized into product of two first order differential operators and the energy spectrum and wave functions of these two Hamiltonians are related to each other by supersymmetric relations [18]. On the other hand, it has been shown that the second order differential equations of mathematical physics and their associated differential equations have the properties of supersymmetry and shape invariance $[19,20]$. By introducing the master function formalism, the authors could transform these equations into Schrodinger equation and obtained some well-known onedimensional shape invariant potentials. The master function formalism as an interesting method can be also used in order to study the relativistic and nonrelativistic quantum systems [21-23]. In [24], the curved Dirac equation in two- and three-dimensional spaces has been studied only in presence of magnetic field. In two-dimensional space, they let an imaginary value for radial component of electromagnetic potential and obtained different angular gauge fields in terms of master function. Now in this work we consider the curved Dirac equation in polar coordinates in the presence of electromagnetic field and show that after inserting a special relation between the components of electromagnetic potential we can obtain a Schrodinger-like second order differential equation 
for the upper spinor component. We then study this equation from master function formalism.

Hence, the paper is organized as follows. In Section 2, we consider the Dirac equation in curved space-time in polar coordinates and construct a Schrodinger-like differential equation for the upper spinor component by using a unitary transformation. In Section 3, we review the factorization formalism of second order differential equations of mathematical physics obtained from the master function. Then, in Section 4, we show that the Schrodinger-like equation obtained from Dirac equation has the shape invariance symmetry in the framework of master function formalism. The electrostatic potential, the energy spectrum, and the wave function of the Dirac equation are obtained in terms of master function and corresponding weight function. After that, we give an example and the other cases of electrostatic potential are given in the Appendix. At the end, we give a brief conclusion in Section 5.

\section{The $(2+1)$ Curved Dirac Equation in Polar Coordinates}

The Dirac equation in Minkowski space-time is $(\hbar=c=1)$ [25]

$$
\left(i \gamma^{a} \partial_{a}-m\right) \psi=0
$$

where the $4 \times 4$ constant matrices $\gamma^{a}$ satisfy the following anticommutation relation in terms of Minkowski metric:

$$
\left\{\gamma^{a}, \gamma^{b}\right\}=2 I \eta^{a b}
$$

Generalization of the Dirac equation from flat to curved space-time needs two modifications. First of all, the $\gamma^{a}$ matrices should be replaced by coordinate dependent matrices $\gamma^{\mu}$ by using bein or tetrad formalism:

$$
\gamma^{\mu}=e_{a}^{\mu} \gamma^{a}
$$

where the tetrads $e^{a}{ }_{\mu}$ and their inverse $e^{\mu}{ }_{a}$ transform the physical quantities from flat to curved frames and vice versa. So the metric tensor of the curved space-time can be related to Minkowski metric by

$$
g_{\mu \nu}=\eta_{a b} e^{a} e^{b}{ }_{\nu}
$$

As the second modification, one should put the covariant derivative instead of partial derivative since the partial derivative of a spinor does not transform like a spinor. The covariant derivative of a spinor has the following form [26]:

$$
\nabla_{\mu} \psi=\partial_{\mu} \psi+\Gamma_{\mu} \psi
$$

where the spinorial affine connection $\Gamma_{\mu}$ can be obtained as

$$
\Gamma_{\mu}=\frac{1}{8} \omega_{\mu c d}\left[\gamma^{c}, \gamma^{d}\right]
$$

The spin connection $\omega_{\mu}{ }^{c}{ }_{d}$ satisfies the following relation:

$$
\omega_{\mu}{ }^{c}{ }_{d}=e^{c}{ }_{\nu} e^{\sigma}{ }_{d} \Gamma_{\sigma \mu}^{\nu}+e^{c}{ }_{\nu} \partial_{\mu} e^{\nu}{ }_{d},
$$

where $\Gamma_{\sigma \mu}^{\nu}$ is the Christoffel symbol in Levi-Civita connection. Therefore, the Dirac equation in curved space-time has the following form:

$$
\left[i \gamma^{\mu}\left(\partial_{\mu}+\frac{1}{8} \omega_{\mu c d}\left[\gamma^{c}, \gamma^{d}\right]\right)-m\right] \psi=0 .
$$

By considering the polar coordinates $(r, \theta)$ the metric tensor in $(2+1)$ dimension can be represented as

$$
g_{\mu \nu}=d t \otimes d t-d r \otimes d r-r^{2} d \theta \otimes d \theta,
$$

and the tetrads corresponding to this metric are as follows:

$$
e_{a}^{\mu}=\left(e_{\mu}^{a}\right)^{-1}=\left(\begin{array}{ccc}
1 & 0 & 0 \\
0 & 1 & 0 \\
0 & 0 & \frac{1}{r}
\end{array}\right) .
$$

The $(2+1)$ Dirac equation in the presence of electromagnetic field according to metric (9) is

$$
\begin{aligned}
& {\left[i \gamma^{\mu}\left(\partial_{\mu}-i e A_{\mu}+\frac{1}{8} \omega_{\mu c d}\left[\gamma^{c}, \gamma^{d}\right]\right)-m\right] \psi(t, r, \theta)} \\
& \quad=0
\end{aligned}
$$

where $A_{\mu}$ are the components of electromagnetic potential. As a convention, the Greek and Latin indices refer to global and local space-time indices which take the values $t, r, \theta$ and $0,1,2$, respectively. In $(2+1)$ dimension, the $4 \times 4$ Dirac matrices can be replaced by Pauli matrices:

$$
\begin{aligned}
& \gamma^{0}=\sigma^{3}, \\
& \gamma^{1}=i \sigma^{2}, \\
& \gamma^{3}=-i \sigma^{1},
\end{aligned}
$$

which satisfy (2). By calculating the nonzero components of Christoffel symbols as

$$
\begin{aligned}
& \Gamma_{\theta \theta}^{r}=-r, \\
& \Gamma_{r \theta}^{\theta}=\frac{1}{r},
\end{aligned}
$$

the nonvanishing components of spin connection can be obtained according to (7) as

$$
\omega_{\theta 12}=-\omega_{\theta 21}=1 \text {. }
$$

By substituting these expressions into (11), we have 


$$
\left(\begin{array}{cc}
i \partial_{t}+e A_{t}-m & i \partial_{r}+\frac{1}{r} \partial_{\theta}-\frac{i e}{r} A_{\theta}+\frac{i}{2 r}+e A_{r} \\
-i \partial_{r}+\frac{1}{r} \partial_{\theta}-\frac{i e}{r} A_{\theta}-\frac{i}{2 r}-e A_{r} & -i \partial_{t}-e A_{t}-m
\end{array}\right) \psi(t, r, \theta)=0
$$

Now, we suppose that the components of the electromagnetic potential only depend on the parameter $r$; hence, the temporal and angular parts of the wave function can be separated as

$$
\begin{aligned}
& \psi(t, r, \theta) \\
& \quad=\exp \left[i\left(k \theta-E t+e \int A_{r} d r\right)\right] r^{-1 / 2}\left(\begin{array}{c}
\varphi_{1}(r) \\
i \varphi_{2}(r)
\end{array}\right),
\end{aligned}
$$

where the time evolution of the wave function is considered as $\exp (-i E t)$ and $k$ is a constant. In (16), the terms $r^{-1 / 2}$ and $\exp \left(e \int A_{r} d r\right)$ are used in order to eliminate the factors $\pm\left(i / 2 r+e A_{r}\right)$ from the off-diagonal elements of matrix (15). Replacing this expression in (15) yields

$$
\begin{aligned}
& \left(\begin{array}{cc}
-e A_{t}+m & \frac{d}{d r}+\frac{k}{r}-\frac{e}{r} A_{\theta} \\
-\frac{d}{d r}+\frac{k}{r}-\frac{e}{r} A_{\theta} & -e A_{t}-m
\end{array}\right)\left(\begin{array}{l}
\varphi_{1}(r) \\
\varphi_{2}(r)
\end{array}\right) \\
& =E\left(\begin{array}{l}
\varphi_{1}(r) \\
\varphi_{2}(r)
\end{array}\right) .
\end{aligned}
$$

As we see, the off-diagonal elements of the above matrix are well-known operators in supersymmetric quantum mechanics. This equation gives two coupled first order differential equations for two radial spinor components $\varphi_{1}(r)$ and $\varphi_{2}(r)$. Our calculations show that, by eliminating the lower or upper component, one can obtain a second order differential equation in terms of another which includes first order derivative. In order to get a Schrodinger-like differential equation, let us use the following unitary transformation introduced in $[27,28]$

$$
\left(\begin{array}{l}
\varphi_{1}(r) \\
\varphi_{2}(r)
\end{array}\right)=\left(\begin{array}{cc}
\cos \rho & \sin \rho \\
-\sin \rho & \cos \rho
\end{array}\right)\left(\begin{array}{c}
F(r) \\
G(r)
\end{array}\right)
$$

where $\rho$ is a constant. After calculation, we find that the necessary condition that vanishes the first order derivative is as follows:

$$
e A_{t}+m \cos 2 \rho-\frac{\sin 2 \rho}{r}\left(k-e A_{\theta}\right)+E=\text { constant. }
$$

By letting this constant as $E+m \cos 2 \rho$, we have

$$
A_{\theta}=\frac{k}{e}-\frac{r}{\sin 2 \rho} A_{t} \text {. }
$$

This relation between the components of electromagnetic potential enables us to eliminate $A_{\theta}$ in (17) and write it only in terms of $A_{t}$. Therefore, by letting (18) and (20) into (17), we get

$$
\begin{aligned}
& \left(-2 e A_{t}+m \cos 2 \rho-E\right) F+\frac{d G}{d r} \\
& +\left(m \sin 2 \rho+\frac{e}{T} A_{t}\right) G=0 \\
& \left(m \sin 2 \rho+\frac{e}{T} A_{t}\right) F-\frac{d F}{d r}-(m \cos 2 \rho+E) G=0
\end{aligned}
$$

where $T=\tan 2 \rho$. By using these equations, we obtain the following Schrodinger-like second order differential equation for $F(r)$ :

$$
\begin{aligned}
& -\frac{d^{2} F}{d r^{2}}+\left(\frac{e}{T} \frac{d A_{t}}{d r}-2 e E A_{t}+\frac{e^{2} A_{t}^{2}}{T^{2}}-E^{2}+m^{2}\right) F \\
& =0 .
\end{aligned}
$$

Also the lower component $G(r)$ can be written in terms of upper component as

$$
\begin{aligned}
& G(r) \\
& \quad=\frac{1}{E+m \cos 2 \rho}\left(-\frac{d}{d r}+m \sin 2 \rho+\frac{e A_{t}}{T}\right) F(r) .
\end{aligned}
$$

In [29], we have solved (22) for two different kinds of electrostatic potentials. Here we want to extend our work and give general formalism in order to solve this equation for different kinds of electrostatic potentials.

It is easy to see that the second term of (22) can be written as Riccati equation, and so using the properties of supersymmetry in quantum mechanics, the electrostatic potential $A_{t}$ can be related to super partner of solvable potentials. Hence, we want to find the relevant choices for $A_{t}$, such that (22) can be factorized into the product of two first order differential operators. Of course, we use the master function formalism introduced in $[19,20]$ and try to relate the shape invariance and supersymmetric properties of second order differential equations of mathematical physics to our work. So, in the next section, we first review this formalism briefly.

\section{Shape Invariance Property of Second Order Differential Equations of Mathematical Physics: Master Function Approach}

The general form of second order differential equation can be written in terms of master function $A(x)$ which is 
a polynomial at most order two and corresponding weight function $W(x)$ as [20]

$$
\begin{aligned}
& A(x) \varphi_{n}^{\prime \prime}(x)+\frac{(A(x) W(x))^{\prime}}{W(x)} \varphi_{n}^{\prime}(x) \\
& \quad-\left[n\left(\frac{A(x) W^{\prime}(x)}{W(x)}\right)^{\prime}+\frac{n(n+1)}{2} A^{\prime \prime}(x)\right] \varphi_{n}(x) \\
& \quad=0
\end{aligned}
$$

where the nonnegative weight function $W(x)$ at the interval $[a, b]$ is calculated so that $(1 / W(x))(d / d x)(A(x) W(x))$ becomes at most first order polynomial. The interval $[a, b]$ is appointed in this manner that $A(a) W(a)=A(b) W(b)=$ 0 . The polynomials $\varphi_{n}(x)$ have the following Rodrigues representation:

$$
\varphi_{n}(x)=\frac{a_{n}}{W(x)}\left(\frac{d}{d x}\right)^{n}\left(A^{n}(x) W(x)\right) .
$$

By introducing two first order differential operators

$$
\begin{aligned}
& \widetilde{B}(n)=A(x) \frac{d}{d x}+\frac{n}{2} A^{\prime}(x)+\frac{A(x) W^{\prime}(x)}{W(x)}+n \\
& \cdot \frac{A^{\prime}(0)\left(A(x) W^{\prime}(x) / W(x)\right)^{\prime}-A^{\prime \prime}(x)\left(A W^{\prime} / W\right)(0)}{2\left[\left(A(x) W^{\prime}(x) / W(x)\right)^{\prime}+n A^{\prime \prime}(x)\right]}, \\
& \widetilde{A}(n)=-A(x) \frac{d}{d x}+\frac{n}{2} A^{\prime}(x)+n \\
& \cdot \frac{A^{\prime}(0)\left(A(x) W^{\prime}(x) / W(x)\right)^{\prime}-A^{\prime \prime}(x)\left(A W^{\prime} / W\right)(0)}{2\left[\left(A(x) W^{\prime}(x) / W(x)\right)^{\prime}+n A^{\prime \prime}(x)\right]}
\end{aligned}
$$

(24) can be factorized in a shape invariant form with respect to quantum number $n$ :

$$
\begin{aligned}
\widetilde{B}(n) \widetilde{A}(n) \varphi_{n}(x) & =E(n) \varphi_{n}(x), \\
\widetilde{A}(n) \widetilde{B}(n) \varphi_{n-1}(x) & =E(n) \varphi_{n-1}(x),
\end{aligned}
$$

where the energy spectrum is given by

$$
\begin{aligned}
& E(n) \\
& =\frac{n}{4\left[\left(A(x) W^{\prime}(x) / W(x)\right)^{\prime}+n A^{\prime \prime}(x)\right]^{2}}\left\{4\left(\frac{A(x) W^{\prime}(x)}{W(x)}\right)^{\prime 2}\right. \\
& \cdot\left[n A^{\prime 2}(0)-A(0)\left(\frac{A(x) W^{\prime}(x)}{W(x)}\right)^{\prime}\right]-\left(\frac{A W^{\prime}}{W}\right)(0) \\
& \cdot\left[A^{\prime \prime}(x)\left(\frac{A W^{\prime}}{W}\right)(0)-2 A^{\prime}(0)\left(\frac{A(x) W^{\prime}(x)}{W(x)}\right)^{\prime}\right] \\
& \cdot\left[2\left(\frac{A(x) W^{\prime}(x)}{W(x)}\right)^{\prime}+n A^{\prime \prime}(x)\right]+n^{2} A^{\prime \prime}(0) \\
& \cdot\left(A^{\prime 2}(0)-2 A^{\prime \prime}(0) A(0)\right)\left[n A^{\prime \prime}(0)+4\left(\frac{A(x) W^{\prime}(x)}{W(x)}\right)^{\prime}\right] \\
& \left.-10 n A(0) A^{\prime \prime}(x)\left(\frac{A(x) W^{\prime}(x)}{W(x)}\right)^{\prime 2}\right\} \text {. }
\end{aligned}
$$

From (27), it is evident that $\widetilde{B}(n)$ and $\widetilde{A}(n)$, as the raising and lowering operators, respectively, satisfy the following relations:

$$
\begin{aligned}
\widetilde{B}(n) \varphi_{n-1}(x) & =\sqrt{E(n)} \varphi_{n}(x), \\
\widetilde{A}(n) \varphi_{n}(x) & =\sqrt{E(n)} \varphi_{n-1}(x) .
\end{aligned}
$$

As we see, the operators $\widetilde{B}(n)$ and $\widetilde{A}(n)$ in (26) are not the differential supersymmetric self-adjoint operators. By inserting a similarity transformation and a change of variable as

$$
\begin{aligned}
A(n) & =W^{1 / 2}(x) \widetilde{B}(n) W^{-1 / 2}(x), \\
A^{\dagger}(n) & =W^{1 / 2}(x) \widetilde{A}(n) W^{-1 / 2}(x), \\
\frac{d r}{d x} & =\frac{1}{A(x)},
\end{aligned}
$$

(27) can be rewritten as the following form:

$$
\begin{gathered}
A(n) A^{\dagger}(n) \psi_{n}(r)=E(n) \psi_{n}(r), \\
A^{\dagger}(n) A(n) \psi_{n-1}(r)=E(n) \psi_{n-1}(r) .
\end{gathered}
$$

The new self-adjoint operators $A(n)$ and $A^{\dagger}(n)$ are given by

$$
\begin{gathered}
A(n)=\frac{d}{d r}+w_{n}(r), \\
A^{\dagger}(n)=-\frac{d}{d r}+w_{n}(r),
\end{gathered}
$$

where the superpotential $w_{n}(r)$ can be determined as

$$
\begin{aligned}
& w_{n}(r)=\frac{1}{2}\left[n A^{\prime}(x)+\frac{A(x) W^{\prime}(x)}{W(x)}+n\right. \\
& \left.\cdot \frac{A^{\prime}(0)\left(A(x) W^{\prime}(x) / W(x)\right)^{\prime}-A^{\prime \prime}(x)\left(A W^{\prime} / W\right)(0)}{\left(A(x) W^{\prime}(x) / W(x)\right)^{\prime}+n A^{\prime \prime}(x)}\right]_{x=x(r)}
\end{aligned}
$$

and the wave function $\psi_{n}(r)$ can be obtained by product of $W^{1 / 2}(x)$ into orthogonal polynomials $\varphi_{n}(x)$ as

$$
\begin{aligned}
\psi_{n}(r) & =W^{1 / 2}(x) \varphi_{n}(x) \\
& =\left.\frac{a_{n}}{W^{1 / 2}(x)}\left(\frac{d}{d x}\right)^{n}\left(A^{n}(x) W(x)\right)\right|_{x=x(r)} .
\end{aligned}
$$

Also from (29), (30), and (36), one can get

$$
\begin{aligned}
& A(n) \psi_{n-1}(r)=\sqrt{E(n)} \psi_{n}(r), \\
& A^{\dagger}(n) \psi_{n}(r)=\sqrt{E(n)} \psi_{n-1}(r) .
\end{aligned}
$$




\section{Solution of the Curved Dirac Equation via Master Function Approach}

In this section, we want to determine the electrostatic potential such that (22) becomes Schrodinger equation with some shape invariant potentials. As a result, the relativistic energy and wave functions of the curved Dirac equation can be obtained in terms of nonrelativistic energy and wave functions. For this aim, we insert the operators $A(n)$ and $A^{\dagger}(n)$ given in (34) into Schrodinger equation (32) and get

$$
\left[-\frac{d^{2}}{d r^{2}}+w_{n}^{2}(r)+\frac{d w_{n}(r)}{d r}\right] \psi_{n}(r)=E(n) \psi_{n}(r) .
$$

Let us compare (22) with the above Schrodinger equation derived from master function formalism

$$
\begin{aligned}
w_{n}^{2}(r) & +\frac{d w_{n}(r)}{d r}-E(n) \\
& =\frac{e}{T} \frac{d A_{t}}{d r}-2 e E A_{t}+\frac{e^{2} A_{t}^{2}}{T^{2}}-E^{2}+m^{2} .
\end{aligned}
$$

Solving this equation gives the electrostatic potential and relativistic energy as

$$
\begin{aligned}
A_{t} & =\frac{T}{e}\left(w_{n}(r)+E T\right), \\
E & =\cos 2 \rho\left[E(n)+m^{2}\right]^{1 / 2} .
\end{aligned}
$$

Therefore, by letting different choices for master function $A(x)$ and weight function $W(x)$, we specify the superpotential and nonrelativistic energy according to (35) and (28); then, by substituting them into (40) and (41), we obtain the electrostatic potential and relativistic energy which satisfy the Dirac equation (17). The upper spinor component is determined in terms of Rodrigues representation of orthogonal polynomials from (36). Also, we can calculate the lower component of the wave function from (23) and (37) as

$$
\begin{aligned}
& G(r)=\frac{1}{E+m \cos 2 \rho}\left(\sqrt{E(n)} \psi_{n-1}(r)\right. \\
& \left.+(m \sin 2 \rho+E T) \psi_{n}(r)\right) .
\end{aligned}
$$

Finally, according to condition (20), the angular part of electromagnetic potential is obtained as

$$
A_{\theta}=\frac{k}{e}-\frac{r}{\sin 2 \rho} \frac{T}{e}\left(w_{n}(r)+E T\right) .
$$

On the other hand, we can specify the electromagnetic tensor. It is well known that the electromagnetic potential is a one form [30]

$$
\begin{aligned}
A= & A_{\mu} d x^{\mu}=A_{t} d t+A_{r} d r+A_{\theta} d \theta \\
= & \frac{T}{e}\left(w_{n}(r)+E T\right) d t+A_{r}(r) d r \\
& +\left(\frac{k}{e}-\frac{r}{\sin 2 \rho} \frac{T}{e}\left(w_{n}(r)+E T\right)\right) d \theta .
\end{aligned}
$$

The electromagnetic tensor is also defined as the exterior derivative of electromagnetic tensor:

$$
\begin{aligned}
F= & d A \\
= & \frac{T}{e} \frac{d w_{n}(r)}{d r} d r \wedge d t \\
& -\frac{1}{\sin 2 \rho} \frac{T}{e}\left[w_{n}(r)+E T+r \frac{d w_{n}(r)}{d r}\right] d r \wedge d \theta .
\end{aligned}
$$

It is obvious that the radial part of electromagnetic potential $A_{r}$ does not contribute to the magnetic field because of its radial symmetry.

In the following, we apply this formalism for a special case of master function and the other cases are given in the Appendix.

Example 1. Let $A(x)=x$. Then, taking into consideration that $(1 / W(x))(d / d x)(A(x) W(x))$ is at most first order polynomial, the weight function $W(x)$ can be obtained as

$$
W(x)=x^{\alpha} e^{-\beta x}, \quad 0 \leq x<+\infty, \alpha>-1, \beta>0 .
$$

Using (35), the superpotential is determined as follows:

$$
w_{n}(r)=\frac{1}{2}(2 n+\alpha-\beta x),
$$

where by changing the variable $x=e^{r}$, according to (31), it leads to superpotential of the Morse potential [31, 32]. Now we determine the electrostatic potential of the Dirac equation from (40)

$$
A_{t}=\frac{T}{e}\left[\frac{1}{2}\left(2 n+\alpha-\beta e^{r}\right)+E T\right] .
$$

Substituting this expression into (22) yields

$$
\begin{aligned}
& -\frac{d^{2} F(r)}{d r^{2}}+\left(\frac{\beta^{2}}{4} e^{2 r}-\frac{\beta}{2} e^{r}(1+2 n+\alpha)+\frac{1}{4}(2 n+\alpha)^{2}\right. \\
& \left.-E^{2}\left(T^{2}+1\right)+m^{2}\right) F(r)=0,
\end{aligned}
$$

where by letting the relativistic energy according to (41) and (28)

$$
E=\cos 2 \rho\left[n(n+\alpha)+m^{2}\right]^{1 / 2}
$$

then it can be factorized as the product of raising and lowering operators

$$
\begin{aligned}
A(n) A^{\dagger}(n) F_{n}(r) & =n(n+\alpha) F_{n}(r), \\
A^{\dagger}(n) A(n) F_{n-1}(r) & =n(n+\alpha) F_{n-1}(r),
\end{aligned}
$$

where

$$
\begin{aligned}
A(n) & =\frac{d}{d r}+\frac{1}{2}\left(2 n+\alpha-\beta e^{r}\right), \\
A^{\dagger}(n) & =-\frac{d}{d r}+\frac{1}{2}\left(2 n+\alpha-\beta e^{r}\right) .
\end{aligned}
$$


Introducing a new function according to (36) as

$$
F_{n}(x(r))=x^{\alpha / 2} e^{-(\beta / 2) x} \varphi_{n}(x(r)),
$$

and changing variable $d r / d x=1 / x,(49)$ is transformed into the following differential equation:

$$
x \varphi_{n}^{\prime \prime}(x)+[(\alpha+1)-\beta x] \varphi_{n}^{\prime}(x)+n \beta \varphi_{n}(x)=0,
$$

which is the associated Laguerre differential equation where $\varphi_{n}(x)$ is specified from (25) as

$$
\varphi_{n}(x)=a_{n} x^{-\alpha} e^{\beta x}\left(\frac{d}{d x}\right)^{n}\left(x^{n+\alpha} e^{-\beta x}\right) .
$$

Also from (42), we get

$$
\begin{aligned}
& G(x(r))=\frac{1}{E+m \cos 2 \rho}\left[\sqrt{n(n+\alpha)} F_{n-1}(x(r))\right. \\
& \left.\quad+(m \sin 2 \rho+E T) F_{n}(x(r))\right] .
\end{aligned}
$$

According to condition (43), we have the following relation for angular component of electromagnetic potential:

$$
A_{\theta}=\frac{k}{e}-\frac{r}{\sin 2 \rho} \frac{T}{e}\left(\frac{1}{2}\left(2 n+\alpha-\beta e^{r}\right)+E T\right) .
$$

By knowing the electromagnetic potential as a one form satisfies (44), we can calculate the electromagnetic tensor as

$$
\begin{aligned}
F= & -\frac{T \beta}{2 e} e^{r} d r \wedge d t \\
& -\frac{T}{e \sin 2 \rho}\left[\frac{1}{2}\left(2 n+\alpha-\beta e^{r}\right)+E T-\frac{\beta}{2} r e^{r}\right] d r
\end{aligned}
$$

$\wedge d \theta$.

\section{Conclusion}

In this paper, we have studied the $(2+1)$ Dirac equation in curved space-time in the presence of both electric and magnetic fields. We have tried to transform this equation into a Schrodinger-like equation for the upper spinor component by applying a unitary transformation and constraint between the components of the gauge field. We have also proposed the electrostatic potentials and relativistic energies in such a way that this equation was transformed into second order differential equations of mathematical physics in master function formalism. The spinor wave function is also specified in terms of Rodrigues polynomials related to each differential equation.

\section{Appendix}

Here, we list some choices for master function and corresponding weight function. For each case, similar to given example in this paper, the components of gauge field, electromagnetic tensor, energy spectra, and spinor wave functions for the curved Dirac equation in polar coordinates have been obtained and in the following we give them briefly:

$$
\begin{aligned}
& A(x)=1, \\
& W(x)=e^{-(1 / 2) \alpha x^{2}+\beta x} \quad-\infty<x<+\infty, \alpha>0,-\infty<\beta<+\infty, \\
& r=x, \\
& A_{t}=\frac{T}{e}\left(-\frac{\alpha}{2} r+\frac{\beta}{2}+E T\right), \\
& A_{\theta}=\frac{k}{e}-\frac{T}{e \sin 2 \rho} r\left(-\frac{\alpha}{2} r+\frac{\beta}{2}+E T\right), \\
& F=-\frac{\alpha T}{2 e} d r \wedge d t-\frac{T}{e \sin 2 \rho}\left(-\alpha r+\frac{\beta}{2}+E T\right) d r \wedge d \theta, \\
& E=\cos 2 \rho\left[n \alpha+m^{2}\right]^{1 / 2} \\
& F_{n}(x(r))=a_{n} e^{(1 / 4) \alpha x^{2}-(\beta / 2) x}\left(\frac{d}{d x}\right)^{n}\left(e^{-(1 / 2) \alpha x^{2}+\beta x}\right), \\
& a_{n}=\frac{a_{n-1}}{\sqrt{n \alpha}} \\
& G(x(r))=\frac{1}{E+m \cos 2 \rho}\left(\sqrt{n \alpha} F_{n-1}(x(r))\right. \\
& \left.+(m \sin 2 \rho+E T) F_{n}(x(r))\right) . \\
& A(x)=x, \\
& W(x)=x^{\alpha} e^{-\beta x}, \quad 0 \leq x<+\infty, \alpha>-1, \beta>0, \\
& x=e^{r}, \\
& A_{t}=\frac{T}{e}\left[\frac{1}{2}\left(2 n+\alpha-\beta e^{r}\right)+E T\right] \\
& A_{\theta}=\frac{k}{e}-\frac{T}{e \sin 2 \rho} r\left[\frac{1}{2}\left(2 n+\alpha-\beta e^{r}\right)+E T\right], \\
& E=\cos 2 \rho\left[n(n+\alpha)+m^{2}\right]^{1 / 2} \text {, } \\
& F=-\frac{T \beta}{2 e} e^{r} d r \wedge d t-\frac{T}{e \sin 2 \rho}\left[\frac{1}{2}\left(2 n+\alpha-\beta e^{r}\right)+E T-\frac{\beta}{2} r e^{r}\right] d r \\
& \wedge d \theta, \\
& F_{n}(x(r))=a_{n} x^{-\alpha / 2} e^{(\beta / 2) x}\left(\frac{d}{d x}\right)^{n}\left(x^{n+\alpha} e^{-\beta x}\right), \\
& a_{n}=\frac{a_{n-1}}{\sqrt{n(n+\alpha)}} \\
& G(x(r))=\frac{1}{E+m \cos 2 \rho}\left[\sqrt{n(n+\alpha)} F_{n-1}(x(r))\right. \\
& \left.+(m \sin 2 \rho+E T) F_{n}(x(r))\right], \\
& A(x)=1-x^{2}, \\
& W(x)=(1-x)^{\alpha}(1+x)^{\beta}, \quad-1<x<+1, \alpha>-1, \beta>-1, \\
& x=\tanh r \text {, } \\
& A_{t}=\frac{T}{e}\left[\frac{-2 n-\alpha-\beta}{2} \tanh r+\frac{\beta^{2}-\alpha^{2}}{2(2 n+\alpha+\beta)}+E T\right], \\
& A_{\theta}=\frac{k}{e}-\frac{T}{e \sin 2 \rho} r\left[\frac{-2 n-\alpha-\beta}{2} \tanh r+\frac{\beta^{2}-\alpha^{2}}{2(2 n+\alpha+\beta)}\right. \\
& +E T]
\end{aligned}
$$




$$
\begin{aligned}
& E=\cos 2 \rho\left[\frac{4 n(n+\alpha)(n+\beta)(n+\alpha+\beta)}{(2 n+\alpha+\beta)^{2}}+m^{2}\right]^{1 / 2} \\
& F=\frac{-T(2 n+\alpha+\beta)}{2 e} \frac{1}{\cosh ^{2} r} d r \wedge d t \\
&-\frac{T}{e \sin 2 \rho}\left[\frac{-2 n-\alpha-\beta}{2} \tanh r+\frac{\beta^{2}-\alpha^{2}}{2(2 n+\alpha+\beta)}+E T\right. \\
&\left.+\frac{-2 n-\alpha-\beta}{2} \frac{r}{\cosh ^{2} r}\right] d r \wedge d \theta, \\
& F_{n}(x(r))=a_{n}(1-x)^{-\alpha / 2}(1+x)^{-\beta / 2}\left(\frac{d}{d x}\right)^{n} \\
& \quad \cdot\left((1-x)^{n+\alpha}(1+x)^{n+\beta}\right), \quad a_{n}=\frac{1}{2} \sqrt{\frac{n+\alpha+\beta}{n(n+\alpha)(n+\beta)}} a_{n-1}
\end{aligned}
$$$$
G(x(r))
$$$$
=\frac{1}{E+m \cos 2 \rho}\left[\sqrt{\frac{4 n(n+\alpha)(n+\beta)(n+\alpha+\beta)}{(2 n+\alpha+\beta)^{2}}} F_{n-1}(x(r))\right.
$$$$
\left.+(m \sin 2 \rho+E T) F_{n}(x(r))\right] .
$$

$\mathrm{A}(x)=x^{2}-1$,

$W(x)=(x-1)^{\alpha}(x+1)^{\beta}$,

$$
-\infty<x<-1, \beta>-1, \alpha+\beta<-2,
$$

$x=-\operatorname{coth} r$,

$$
\begin{aligned}
A_{t} & =\frac{T}{e}\left[-\frac{2 n+\alpha+\beta}{2} \operatorname{coth} r+\frac{\alpha^{2}-\beta^{2}}{2(2 n+\alpha+\beta)}+E T\right], \\
A_{\theta} & =\frac{k}{e}-\frac{T}{e \sin 2 \rho} r\left[-\frac{2 n+\alpha+\beta}{2} \operatorname{coth} r+\frac{\alpha^{2}-\beta^{2}}{2(2 n+\alpha+\beta)}\right. \\
& +E T],
\end{aligned}
$$$$
E=\cos 2 \rho\left[\frac{4 n(n+\alpha)(n+\beta)(n+\alpha+\beta)}{(2 n+\alpha+\beta)^{2}}+m^{2}\right]^{1 / 2}
$$$$
F=\frac{T(2 n+\alpha+\beta)}{2 e} \frac{1}{\sinh ^{2} r} d r \wedge d t
$$$$
-\frac{T}{e \sin 2 \rho}\left[-\frac{2 n+\alpha+\beta}{2} \operatorname{coth} r+\frac{\alpha^{2}-\beta^{2}}{2(2 n+\alpha+\beta)}+E T\right.
$$$$
\left.+\frac{2 n+\alpha+\beta}{2} \frac{r}{\sinh ^{2} r}\right] d r \wedge d \theta
$$$$
F_{n}(x(r))=a_{n}(x-1)^{-\alpha / 2}(x+1)^{-\beta / 2}\left(\frac{d}{d x}\right)^{n}
$$$$
\cdot\left((x-1)^{n+\alpha}(x+1)^{n+\beta}\right), \quad a_{n}=\frac{1}{2} \sqrt{\frac{n+\alpha+\beta}{n(n+\alpha)(n+\beta)}} a_{n-1}
$$

$G(x(r))$

$$
\begin{aligned}
& =\frac{1}{E+m \cos 2 \rho}\left(\sqrt{\frac{4 n(n+\alpha)(n+\beta)(n+\alpha+\beta)}{(2 n+\alpha+\beta)^{2}}} F_{n-1}(x(r))\right. \\
& \left.+(m \sin 2 \rho+E T) F_{n}(x(r))\right) .
\end{aligned}
$$

$A(x)=x(1-x)$,

$W(x)=x^{\alpha}(1-x)^{\beta}, \quad 0<x<+1, \alpha>-1, \beta>-1$,

$x=\frac{e^{r}}{1+e^{r}}$,

$A_{t}=\frac{T}{e}\left[-\frac{1}{2}(2 n+\alpha+\beta) \frac{e^{r}}{1+e^{r}}\right.$

$$
\left.+\frac{(n+\alpha)(2 n+\alpha+\beta)+(\beta-\alpha) n}{2(2 n+\alpha+\beta)}+E T\right]
$$

$A_{\theta}=\frac{k}{e}-\frac{T}{e \sin 2 \rho} r\left[-\frac{1}{2}(2 n+\alpha+\beta) \frac{e^{r}}{1+e^{r}}\right.$

$$
\left.+\frac{(n+\alpha)(2 n+\alpha+\beta)+(\beta-\alpha) n}{2(2 n+\alpha+\beta)}+E T\right]
$$

$E=\cos 2 \rho\left[\frac{n(n+\alpha)(n+\beta)(n+\alpha+\beta)}{(2 n+\alpha+\beta)^{2}}+m^{2}\right]^{1 / 2}$

$F=-\frac{T}{2 e}(2 n+\alpha+\beta) \frac{e^{r}}{\left(1+e^{r}\right)^{2}} d r \wedge d t$

$-\frac{T}{e \sin 2 \rho}\left[-\frac{1}{2}(2 n+\alpha+\beta) \frac{e^{r}}{1+e^{r}}\right.$

$$
+\frac{(n+\alpha)(2 n+\alpha+\beta)+(\beta-\alpha) n}{2(2 n+\alpha+\beta)}+E T
$$$$
\left.-\frac{1}{2}(2 n+\alpha+\beta) \frac{r e^{r}}{\left(1+e^{r}\right)^{2}}\right] d r \wedge d \theta,
$$

$$
\begin{array}{r}
F_{n}(x(r))=a_{n} x^{-\alpha / 2}(1-x)^{-\beta / 2}\left(\frac{d}{d x}\right)^{n}\left(x^{n+\alpha}(1-x)^{n+\beta}\right), \\
a_{n}=\sqrt{\frac{n+\alpha+\beta}{n(n+\alpha)(n+\beta)}} a_{n-1},
\end{array}
$$

$G(x(r))$

$$
\begin{aligned}
& =\frac{1}{E+m \cos 2 \rho}\left(\sqrt{\frac{n(n+\alpha)(n+\beta)(n+\alpha+\beta)}{(2 n+\alpha+\beta)^{2}}} F_{n-1}(x(r))\right. \\
& \left.+(m \sin 2 \rho+E T) F_{n}(x(r))\right) .
\end{aligned}
$$

\section{Conflict of Interests}

The authors declare that they have no conflict of interests related to this paper.

\section{References}

[1] C. V. Sukumar, "Supersymmetry and the Dirac equation for a central Coulomb field," Journal of Physics. A. Mathematical and General, vol. 18, no. 12, pp. L697-L701, 1985. 
[2] S. M. Ikhdair, "Approximate solutions of the Dirac equation for the Rosen-Morse potential including the spin-orbit centrifugal term," Journal of Mathematical Physics, vol. 51, no. 2, Article ID 023525, 2010.

[3] M. Hamzavi and A. A. Rajabi, "Generalized nuclear WoodsSaxon potential under relativistic spin symmetry limit," ISRN High Energy Physics, vol. 2013, Article ID 987632, 7 pages, 2013.

[4] H. Hassanabadi, E. Maghsoodi, S. Zarrinkamar, and H. Rahimov, "Dirac equation for generalized Pöschl-Teller scalar and vector potentials and a Coulomb tensor interaction by Nikiforov-Uvarv method," Journal of Mathematical Physics, vol. 53, no. 2, Article ID 022104, 2012.

[5] M. Azizi, N. Salehi, and A. A. Rajabi, "Exact solution of the Dirac equation for the Yukawa potential with scalar and vector potentials and tensor interaction," ISRN High Energy Physics, vol. 2013, Article ID 310392, 6 pages, 2013.

[6] S. K. Moayedi and F. Darabi, "Exact solutions of Dirac equation on a 2D gravitational background," Physics Letters A, vol. 322, no. 3-4, pp. 173-178, 2004.

[7] M. Khorrami, M. Alimohammadi, and A. Shariati, "Spin 0 and spin $1 / 2$ quantum relativistic particles in a constant gravitational field," Annals of Physics, vol. 304, no. 2, pp. 91-102, 2003.

[8] V. M. Villalba, "Dirac equation in some homogeneous spacetimes, separation of variables and exact solutions," Modern Physics Letters A. Particles and Fields, Gravitation, Cosmology, Nuclear Physics, vol. 8, no. 25, pp. 2351-2364, 1993.

[9] Y. Sucu and N. Unal, "Exact solution of Dirac equation in $2+1$ dimensional gravity," Journal of Mathematical Physics, vol. 48, no. 5, Article ID 052503, 9 pages, 2007.

[10] A. D. Alhaidari, "Solution of the Dirac equation by separation of variables in spherical coordinates for a large class of non-central electromagnetic potentials," Annals of Physics, vol. 320, no. 2, pp. 453-467, 2005.

[11] V. M. Villalba and E. I. Catalá, "Separation of variables and exact solution of the Klein-Gordon and Dirac equations in an open universe," Journal of Mathematical Physics, vol. 43, no. 10, article 4909, 2002.

[12] X. Zou, L.-Z. Yi, and C.-S. Jia, "Bound states of the Dirac equation with vector and scalar Eckart potentials," Physics Letters A, vol. 346, no. 1-3, pp. 54-64, 2005.

[13] X.-Q. Zhao, C.-S. Jia, and Q.-B. Yang, "Bound states of relativistic particles in the generalized symmetrical double-well potential," Physics Letters, Section A: General, Atomic and Solid State Physics, vol. 337, no. 3, pp. 189-196, 2005.

[14] H. Egrifes and R. Sever, "Bound states of the Dirac equation for the $P T$-symmetric generalized Hulthén potential by the Nikiforov-Uvarov method," Physics Letters A, vol. 344, no. 2-4, pp. 117-126, 2005.

[15] H. Hassanabadi, E. Maghsoodi, A. N. Ikot, and S. Zarrinkamar, "Approximate arbitrary-state solutions of Dirac equation for modified deformed Hylleraas and modified Eckart potentials by the NU method," Applied Mathematics and Computation, vol. 219, no. 17, pp. 9388-9398, 2013.

[16] E. Witten, "Dynamical breaking of supersymmetry," Nuclear Physics B, vol. 188, no. 3, pp. 513-554, 1981.

[17] L. Gendenshtein, "Derivation of exact spectra of the Schrodinger equation by means of supersymmetry," Journal of Experimental and Theoretical Physics Letters, vol. 38, no. 6, pp. 356-359, 1983.

[18] F. Cooper, A. Khare, and U. Sukhatme, "Supersymmetry and quantum mechanics," Physics Reports, vol. 251, no. 5-6, pp. 267385, 1995.
[19] M. A. Jafarizadeh and H. Fakhri, "Parasupersymmetry and shape invariance in differential equations of mathematical physics and quantum mechanics," Annals of Physics, vol. 262, no. 2, pp. 260-276, 1998.

[20] H. Fakhri, "Another bunch of superpotentials in terms of a master function," Physics Letters A, vol. 265, no. 1-2, pp. 20-27, 2000.

[21] H. Fakhri, "Dirac equation for a spin-1/2 charged particle on the 2D sphere $S^{2}$ and the hyperbolic plane $H^{2}$," Journal of Physics $A$. Mathematical and General, vol. 35, no. 30, pp. 6329-6337, 2002.

[22] H. Fakhri, "Solution of the Dirac equation on the homogeneous manifold $S L(2, c) / G L(1, c)$ in the presence of a magnetic monopole field," Journal of Physics A: Mathematical and General, vol. 33, no. 2, pp. 293-305, 2000.

[23] H. Panahi, L. Jahangiri, and S. Asghari Rad, "Generalized Jaynes-Cummings model in master function and supersymmetric representations," The European Physical Journal Plus, vol.129, no. 6, pp. 113-123, 2014.

[24] H. Fakhri and N. Abbasi, "Exact solution of the Dirac equation for a spin-1/2 charged particle in two-dimensional and threedimensional Euclidean spaces with shape invariance symmetry," Journal of Mathematical Physics, vol. 42, no. 6, pp. 24162437, 2001.

[25] W. Greiner, Relativistic Quantum Mechanics, Springer, Berlin, Germany, 1981.

[26] I. D. Lawrie, A Unified Grand Tour of Theoretical Physics, Institute of Physics Publishing, Bristol, UK, 2nd edition, 2002.

[27] A. D. Alhaidari, "Relativistic extension of shape invariant potentials," Journal of Physics A: Mathematical and General, vol. 35, no. 29, pp. 6207-6216, 2002.

[28] A. D. Alhaidari, "Solution of the Dirac equation with positiondependent mass in the Coulomb field," Physics Letters A, vol. 322, no. 1-2, pp. 72-77, 2004.

[29] H. Panahi and L. Jahangiri, "The $(2+1)$ curved Dirac equation in polar coordinates in the presence of electromagnetic field," Annals of Physics, vol. 354, pp. 306-315, 2015.

[30] M. Nakahara, Geometry, Topology and Physics, Institute of Physics, Bristol, UK, 2nd edition, 2003.

[31] J. W. Dabrowska, A. Khare, and U. P. Sukhatme, "Explicit wavefunctions for shape-invariant potentials by operator techniques," Journal of Physics A: Mathematical and General, vol. 21, no. 4, pp. L195-L200, 1988.

[32] G. Levai, "A search for shape-invariant solvable potentials," Journal of Physics A: Mathematical and General, vol. 22, no. 6, pp. 689-702, 1989. 

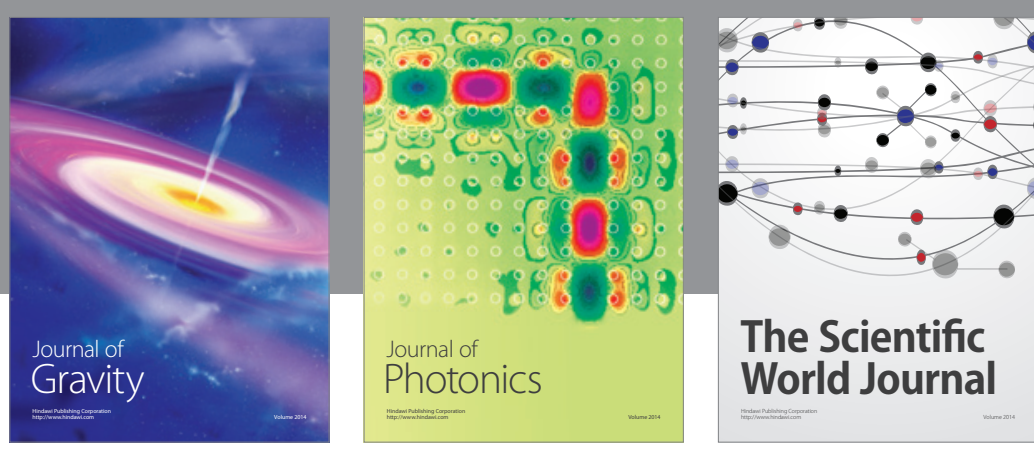

The Scientific World Journal
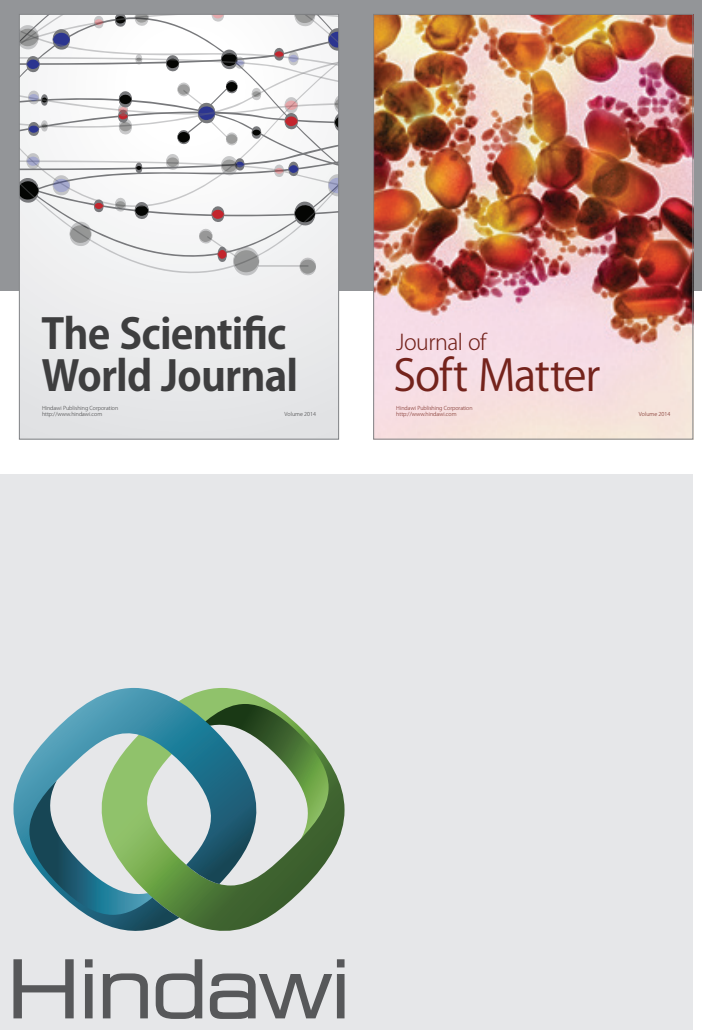

Submit your manuscripts at

http://www.hindawi.com

nternational Journal of

Statistical Mechanics
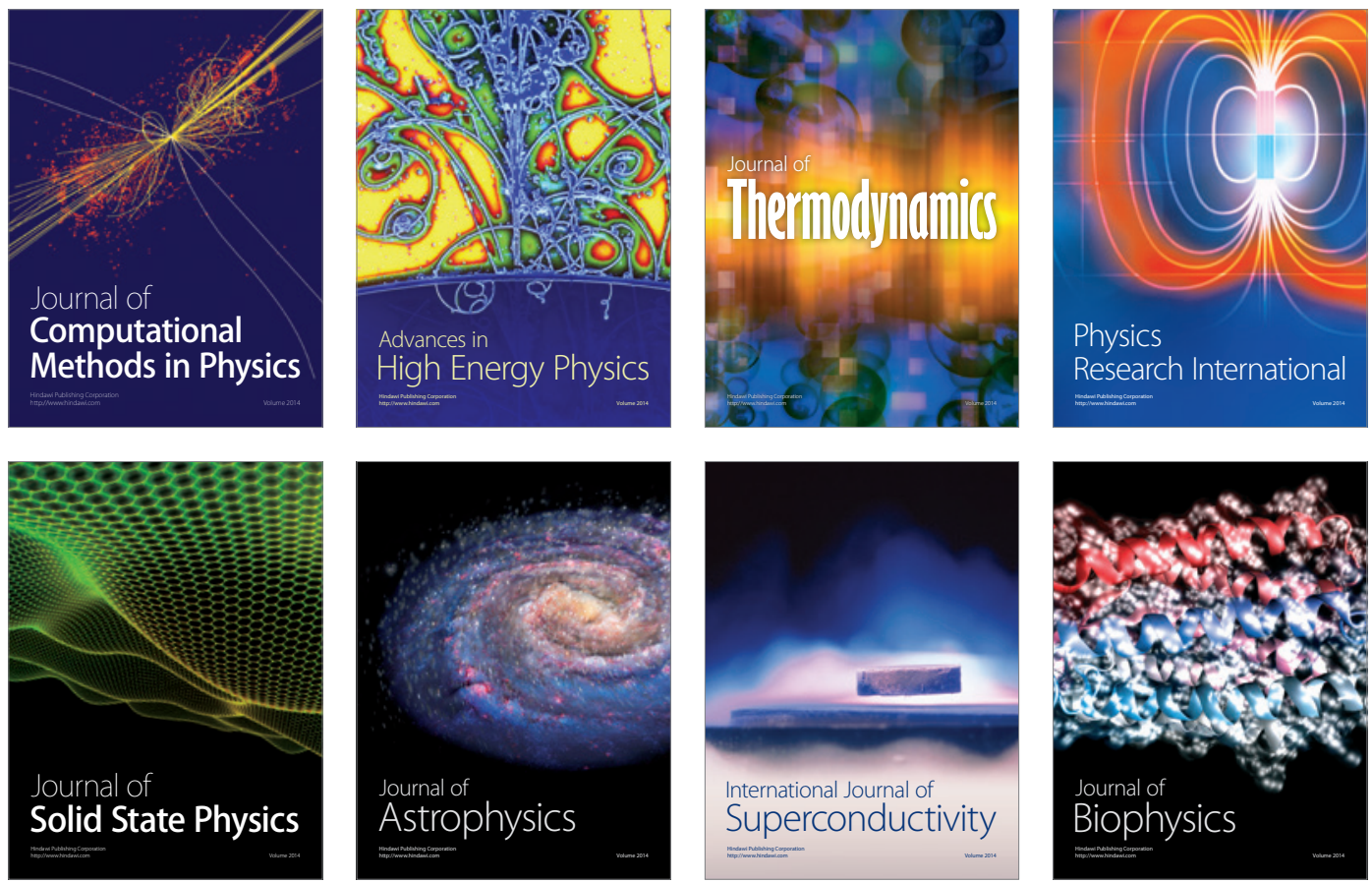
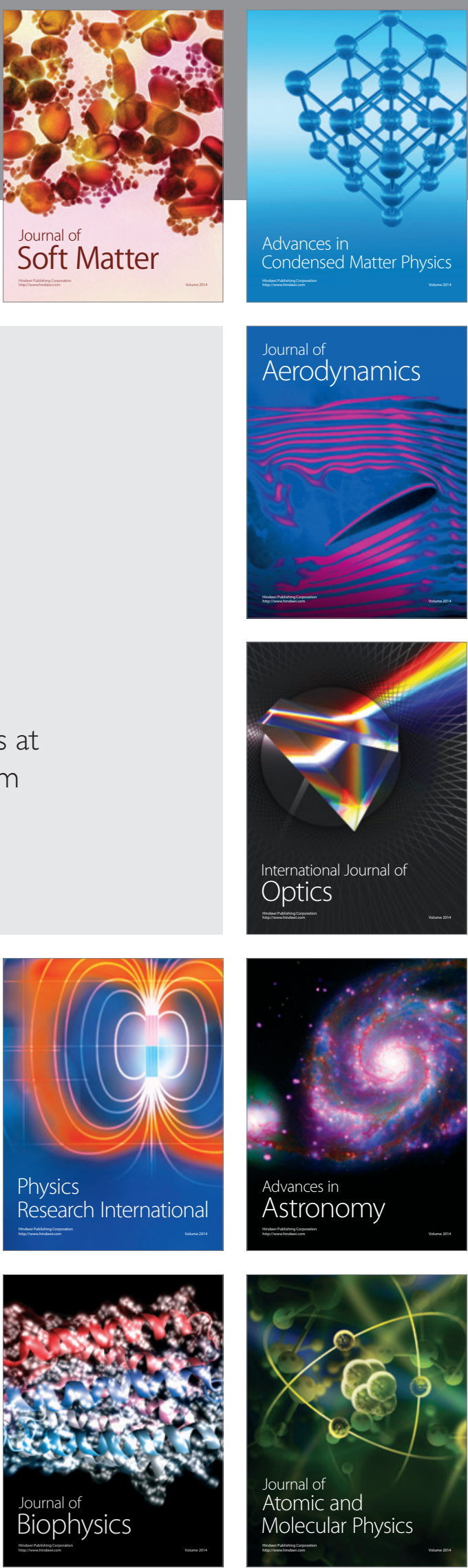